\title{
Strong and $\Delta$-convergence for mixed type total asymptotically nonexpansive mappings in $\operatorname{CAT}(0)$ spaces
}

Shih-sen Chang ${ }^{1 *}$, Lin Wang ${ }^{1}$, Heung Wing Joseph Lee ${ }^{2}$ and Chi-kin Chan ${ }^{2}$

\author{
"Correspondence: \\ changss@yahoo.cn \\ ${ }^{1}$ College of Statistics and \\ Mathematics, Yunnan University of \\ Finance and Economics, Kunming, \\ Yunnan 650221, China \\ Full list of author information is \\ available at the end of the article
}

\begin{abstract}
It is our purpose in this paper first to introduce the class of total asymptotically nonexpansive nonself mappings and to prove the demiclosed principle for such mappings in CAT(0) spaces. Then, a new mixed Agarwal-O'Regan-Sahu type iterative scheme for approximating a common fixed point of two total asymptotically nonexpansive mappings and two total asymptotically nonexpansive nonself mappings is constructed. Under suitable conditions, some strong convergence theorems and $\Delta$-convergence theorems are proved in a CAT(0) space. Our results improve and extend the corresponding results of Agarwal, O'Regan and Sahu (J. Nonlinear Convex Anal. 8(1):61-79, 2007), Guo et al. (Fixed Point Theory Appl. 2012:224, 2012. doi:10.1186/1687-1812-2012-224), Sahin et al. (Fixed Point Theory Appl. 2013:12, 2013. doi:10.1186/1687-1812-2013-12), Chang et al. (Appl. Math. Comput. 219:2611-2617, 2012), Khan and Abbas (Comput. Math. Appl. 61:109-116, 2011), Khan et al. (Nonlinear Anal. 74:783-791, 2011), Xu (Nonlinear Anal., Theory Methods Appl. 16(12):1139-1146, 1991), Chidume et al. (J. Math. Anal. Appl. 280:364-374, 2003) and others.
\end{abstract}

MSC: 47J05; 47H09; 49J25

Keywords: total asymptotically nonexpansive mappings; total asymptotically nonexpansive nonself mappings; CAT(0) space; demiclosed principle; $\Delta$-convergence; strong convergence; mixed Agarwal-O'Regan-Sahu type iterative scheme 
A geodesic space $X$ is a CAT $(0)$ space if for each geodesic triangle $\Delta\left(x_{1}, x_{2}, x_{3}\right)$ in $X$ and its comparison triangle $\bar{\Delta}:=\Delta\left(\overline{x_{1}}, \overline{x_{2}}, \overline{x_{3}}\right)$ in $\mathcal{R}^{2}$, the $\operatorname{CAT}(0)$ inequality

$$
d(x, y) \leq d_{\mathcal{R}^{2}}(\bar{x}, \bar{y})
$$

is satisfied for all $x, y \in \Delta$ and $\bar{x}, \bar{y} \in \bar{\Delta}$.

The initials of the term 'CAT' are in honor of Cartan, Alexanderov and Toponogov. A CAT(0) space is a generalization of the Hadamard manifold, which is a simply connected, complete Riemannian manifold such that the sectional curvature is nonpositive. A thorough discussion of these spaces and their important role in various branches of mathematics are given in [1].

In this paper, we write $(1-t) x \oplus t y$ for the unique point $z$ in the geodesic segment joining from $x$ to $y$ such that

$$
d(z, x)=t d(x, y), d(z, y)=(1-t) d(x, y) .
$$

We also denote by $[x, y]$ the geodesic segment joining from $x$ to $y$, that is, $[x, y]=\{(1-t) x \oplus$ $t y: t \in[0,1]\}$.

A subset $C$ of a $\operatorname{CAT}(0)$ space is convex if $[x, y] \subset C$ for all $x, y \in C$. For elementary facts about CAT(0) spaces, we refer the readers to [1] or [2].

The following lemma plays an important role in our paper.

Lemma 1.1 [2] A geodesic space $X$ is a CAT(0) space if and only if the following inequality holds:

$$
d^{2}((1-t) x \oplus t y, z) \leq(1-t) d^{2}(x, z)+t d^{2}(y, z)-t(1-t) d^{2}(x, y)
$$

for all $x, y, z \in X$ and all $t \in[0,1]$. In particular, if $x, y, z$ are points in a $\mathrm{CAT}(0)$ space and $t \in[0,1]$, then

$$
d((1-t) x \oplus t y, z) \leq(1-t) d(x, z)+t d(y, z) .
$$

Let $(X, d)$ be a metric space, and let $C$ be a nonempty subset of $X$. Recall that $C$ is said to be a retract of $X$ if there exists a continuous map $P: X \rightarrow C$ such that $P x=x, \forall x \in C$. A map $P: X \rightarrow C$ is said to be a retraction if $P^{2}=P$. If $P$ is a retraction, then $P y=y$ for all $y$ in the range of $P$.

A mapping $T: C \rightarrow C$ is said to be nonexpansive if

$$
d(T x, T y) \leq d(x, y), \quad \forall x, y \in C
$$

$T: C \rightarrow C$ is said to be asymptotically nonexpansive if there is a sequence $\left\{k_{n}\right\} \subset[1, \infty)$ with $k_{n} \rightarrow 1$ such that

$$
d\left(T^{n} x, T^{n} y\right) \leq k_{n} d(x, y), \quad \forall n \geq 1, x, y \in C .
$$


$T: C \rightarrow X$ is said to be an asymptotically nonexpansive nonself mapping if there is a sequence $\left\{k_{n}\right\} \subset[1, \infty)$ with $k_{n} \rightarrow 1$ such that

$$
d\left(T(P T)^{n-1} x, T(P T)^{n-1} y\right) \leq k_{n} d(x, y), \quad \forall n \geq 1, x, y \in C,
$$

where $P$ is a nonexpansive retraction of $X$ onto $C$.

$T: C \rightarrow C$ is said to be uniformly L-Lipschitzian if there exists a constant $L>0$ such that

$$
d\left(T^{n} x, T^{n} y\right) \leq L d(x, y), \quad \forall n \geq 1, x, y \in C .
$$

Definition 1.2 A self-mapping $T: C \rightarrow C$ is said to be $\left(\left\{\mu_{n}\right\},\left\{v_{n}\right\}, \zeta\right)$-total asymptotically nonexpansive if there exist nonnegative sequences $\left\{\mu_{n}\right\},\left\{v_{n}\right\}$ with $\mu_{n} \rightarrow 0, v_{n} \rightarrow 0$ and a strictly increasing continuous function $\zeta:[0, \infty) \rightarrow[0, \infty)$ with $\zeta(0)=0$ such that

$$
d\left(T^{n} x, T^{n} y\right) \leq d(x, y)+v_{n} \zeta(d(x, y))+\mu_{n}, \quad \forall n \geq 1, x, y \in C .
$$

Definition 1.3 $T: C \rightarrow X$ is said to be a $\left(\left\{\mu_{n}\right\},\left\{v_{n}\right\}, \zeta\right)$-total asymptotically nonexpansive nonself mapping if there exist nonnegative sequences $\left\{\mu_{n}\right\},\left\{v_{n}\right\}$ with $\mu_{n} \rightarrow 0, v_{n} \rightarrow 0$ and a strictly increasing continuous function $\zeta:[0, \infty) \rightarrow[0, \infty)$ with $\zeta(0)=0$ such that

$$
d\left(T(P T)^{n-1} x, T(P T)^{n-1} y\right) \leq d(x, y)+v_{n} \zeta(d(x, y))+\mu_{n}, \quad \forall n \geq 1, x, y \in C,
$$

where $P$ is a nonexpansive retraction of $X$ onto $C$.

Definition 1.4 A nonself mapping $T: C \rightarrow X$ is said to be uniformly $L$-Lipschitzian if there exists a constant $L>0$ such that

$$
d\left(T(P T)^{n-1} x, T(P T)^{n-1} y\right) \leq L d(x, y), \quad \forall n \geq 1, x, y \in C,
$$

where $P$ is a nonexpansive retraction of $X$ onto $C$.

Remark 1.5 From the definitions, it is to know that each nonexpansive mapping is an asymptotically nonexpansive mapping with a sequence $\left\{k_{n}=1\right\}$, and each asymptotically nonexpansive mapping is a $\left(\left\{\mu_{n}\right\},\left\{v_{n}\right\}, \zeta\right)$-total asymptotically nonexpansive mapping with $\mu_{n}=0, v_{n}=k_{n}-1, n \geq 1$ and $\zeta(t)=t, t \geq 0$.

In 1976, Lim [3] introduced the concept of $\Delta$-convergence in a general metric space. In 2008, Kirk and Panyanak [4] specialized Lim's concept to CAT(0) spaces and proved that it is very similar to the weak convergence in a Banach space setting.

Fixed point theory in a CAT(0) space was first studied by Kirk (see [5, 6]). He showed that every nonexpansive mapping defined on a bounded closed convex subset of a complete $\mathrm{CAT}(0)$ space always has a fixed point. Since then the existence problem of fixed point and the $\Delta$-convergence problem of iterative sequences to a fixed point for nonexpansive mappings, asymptotically nonexpansive mappings in a $\mathrm{CAT}(0)$ space have been rapidly developed and many papers have appeared (see, e.g., [7-26]).

The purpose of this paper is first to introduce the class of total asymptotically nonexpansive nonself mappings and to prove the demiclosed principle for such mappings in $\mathrm{CAT}(0)$ 
spaces. Then, a new mixed Agarwal-O'Regan-Sahu type iterative scheme [27] for approximating a common fixed point of two total asymptotically nonexpansive mappings and two total asymptotically nonexpansive nonself mappings is constructed. Under suitable conditions, some strong convergence theorems and $\Delta$-convergence theorems are proved in a $\mathrm{CAT}(0)$ space. Our results extend and improve the corresponding results of Agarwal, O'Regan and Sahu [27], Guo et al. [28], Sahin [26], Chang et al. [24], Khan and Abbas [22], Khan et al. [23], Chidume et al. [29], Xu [30], Chang et al. [31] and many other recent results.

\section{Demiclosed principle for total asymptotically nonexpansive nonself mappings}

Let $\left\{x_{n}\right\}$ be a bounded sequence in a $\operatorname{CAT}(0)$ space $X$. For $x \in X$, we set

$$
r\left(x,\left\{x_{n}\right\}\right)=\limsup _{n \rightarrow \infty} d\left(x, x_{n}\right) .
$$

The asymptotic radius $r\left(\left\{x_{n}\right\}\right)$ of $\left\{x_{n}\right\}$ is given by

$$
r\left(\left\{x_{n}\right\}\right)=\inf \left\{r\left(x,\left\{x_{n}\right\}\right): x \in X\right\} .
$$

The asymptotic radius $r_{C}\left(\left\{x_{n}\right\}\right)$ of $\left\{x_{n}\right\}$ with respect to $C \subset X$ is given by

$$
r_{C}\left(\left\{x_{n}\right\}\right)=\inf \left\{r\left(x,\left\{x_{n}\right\}\right): x \in C\right\} .
$$

The asymptotic center $A\left(\left\{x_{n}\right\}\right)$ of $\left\{x_{n}\right\}$ is the set

$$
A\left(\left\{x_{n}\right\}\right)=\left\{x \in X: r\left(x,\left\{x_{n}\right\}\right)=r\left(\left\{x_{n}\right\}\right)\right\} .
$$

And the asymptotic center $A_{C}\left(\left\{x_{n}\right\}\right)$ of $\left\{x_{n}\right\}$ with respect to $C \subset X$ is the set

$$
A_{C}\left(\left\{x_{n}\right\}\right)=\left\{x \in C: r\left(x,\left\{x_{n}\right\}\right)=r_{C}\left(\left\{x_{n}\right\}\right)\right\} .
$$

Proposition 2.1 [7] Let $X$ be a complete CAT(0) space, let $\left\{x_{n}\right\}$ be a bounded sequence in $X$ and let $C$ be a closed convex subset of $X$. Then

(1) there exists a unique point $u \in C$ such that

$$
r\left(u,\left\{x_{n}\right\}\right)=\inf _{x \in C} r\left(x,\left\{x_{n}\right\}\right)
$$

(2) $A\left(\left\{x_{n}\right\}\right)$ and $A_{C}\left(\left\{x_{n}\right\}\right)$ both are singleton.

Definition $2.2[3,4]$ Let $X$ be a $\operatorname{CAT}(0)$ space. A sequence $\left\{x_{n}\right\}$ in $X$ is said to $\Delta$-converge to $p \in X$ if $p$ is the unique asymptotic center of $\left\{u_{n}\right\}$ for each subsequence $\left\{u_{n}\right\}$ of $\left\{x_{n}\right\}$. In this case, we write $\Delta-\lim _{n \rightarrow \infty} x_{n}=p$ and call $p$ the $\Delta$-limit of $\left\{x_{n}\right\}$.

\section{Lemma 2.3}

(1) Let $X$ be a complete $\mathrm{CAT}(0)$ space, let $C$ be a closed convex subset of $X$. If $\left\{x_{n}\right\}$ is a bounded sequence in $C$, then the asymptotic center of $\left\{x_{n}\right\}$ is in $C$ [8]; 
(2) Every bounded sequence in a complete CAT(0) space always has a $\Delta$-convergent subsequence [4].

Remark 2.4 Let $X$ be a CAT(0) space and let $C$ be a closed convex subset of $X$. Let $\left\{x_{n}\right\}$ be a bounded sequence in $C$. In what follows, we denote it by

$$
\left\{x_{n}\right\} \rightarrow w \quad \Leftrightarrow \quad \Phi(w)=\inf _{x \in C} \Phi(x)
$$

where $\Phi(x):=\limsup _{n \rightarrow \infty} d\left(x_{n}, x\right)$.

Now we give a connection between the ' $\rightarrow$ ' convergence and $\Delta$-convergence.

Proposition 2.5 Let $X$ be a CAT(0) space, let $C$ be a closed convex subset of $X$ and let $\left\{x_{n}\right\}$ be a bounded sequence in $C$. Then $\Delta-\lim _{n \rightarrow \infty} x_{n}=p$ implies that $\left\{x_{n}\right\} \rightarrow p$.

Proof In fact, if $\Delta-\lim _{n \rightarrow \infty} x_{n}=p$, then it follows from Lemma 2.3 that $p \in C$. Since $A\left(\left\{x_{n}\right\}\right)=\{p\}$, we have $r\left(\left\{x_{n}\right\}\right)=r\left(p,\left\{x_{n}\right\}\right)$. This implies that $\Phi(p)=\inf _{y \in C} \Phi(y)$, i.e., $\left\{x_{n}\right\} \rightarrow p$. The desired conclusion is obtained.

It is well known that one of the fundamental and celebrated results in the theory of nonexpansive mappings is Browder's demiclosed principle [32] which states that if $X$ is a uniformly convex Banach space, $C$ is a nonempty closed convex subset of $X$, and $T: C \rightarrow X$ is a nonexpansive mapping, then $I-T$ is demiclosed at 0 , i.e., for any sequence $\left\{x_{n}\right\}$ in $C$ if $x_{n} \rightarrow x$ weakly and $\left\|(I-T) x_{n}\right\| \rightarrow 0$, then $x=T x$.

Later, $\mathrm{Xu}$ [30] and Chang et al. [31] proved the demiclosed principle for asymptotically nonexpansive mappings in a uniformly convex Banach space. In 2003, Chidume et al. [29] proved the demiclosed principle for asymptotically nonexpansive nonself mappings in uniformly convex Banach spaces.

In this section, by using the convergence ' $\rightarrow$ ' defined by (2.5), we prove the demiclosed principle for total asymptotically nonexpansive nonself mappings in CAT $(0)$ spaces, which extends the results of $\mathrm{Xu}$ [30], Chang et al. [31] and Chidume et al. [29] to CAT(0) spaces.

Theorem 2.6 (Demiclosed principle for total asymptotically nonexpansive nonself mappings in CAT(0) spaces) Let $C$ be a nonempty closed and convex subset of a complete CAT(0) space $X$, and let $T: C \rightarrow X$ be a uniformly L-Lipschitzian and $\left(\left\{\mu_{n}\right\},\left\{v_{n}\right\}, \zeta\right)$-total asymptotically nonexpansive nonself mapping. Let $\left\{x_{n}\right\}$ be a bounded sequence in $C$ such that $\left\{x_{n}\right\} \rightarrow p$ defined by $(2.5)$ and $\lim _{n \rightarrow \infty} d\left(x_{n}, T x_{n}\right)=0$. Then $T p=p$.

Proof By the definition and Proposition 2.1, $\left\{x_{n}\right\} \rightarrow p$ if and only if $A_{C}\left(\left\{x_{n}\right\}\right)=\{p\}$. By Lemma 2.3, we have $A\left(\left\{x_{n}\right\}\right)=\{p\}$.

Since $\lim _{n \rightarrow \infty} d\left(x_{n}, T x_{n}\right)=0$, by induction we can prove that

$$
\lim _{n \rightarrow \infty} d\left(x_{n}, T(P T)^{m-1} x_{n}\right)=0 \quad \text { for each } m \geq 1
$$

In fact, it is obvious that the conclusion is true for $m=1$. Suppose the conclusion holds for $m \geq 1$, now we prove that the conclusion is also true for $m+1$. 
Indeed, since $x_{n} \in C$, we have $x_{n}=P x_{n}$. In addition, since $T$ is uniformly $L$-Lipschitzian, we have

$$
\begin{aligned}
d\left(x_{n}, T(P T)^{m} x_{n}\right) & \leq d\left(x_{n}, T(P T)^{m-1} x_{n}\right)+d\left(T(P T)^{m-1} x_{n}, T(P T)^{m} x_{n}\right) \\
& \leq d\left(x_{n}, T(P T)^{m-1} x_{n}\right)+L d\left(x_{n}, P T x_{n}\right) \\
& =d\left(x_{n}, T(P T)^{m-1} x_{n}\right)+L d\left(P x_{n}, P T x_{n}\right) \\
& \leq d\left(x_{n}, T(P T)^{m-1} x_{n}\right)+L d\left(x_{n}, T x_{n}\right) \rightarrow 0 \quad(\text { as } n \rightarrow \infty) .
\end{aligned}
$$

Equation (2.6) is proved. Hence for each $x \in X$ and $m \geq 1$, we have

$$
\Phi(x):=\limsup _{n \rightarrow \infty} d\left(x_{n}, x\right)=\limsup _{n \rightarrow \infty} d\left(T(P T)^{m-1}\left(x_{n}\right), x\right)
$$

In (2.7), taking $x=T(P T)^{m-1} p, m \geq 1$, we have

$$
\begin{aligned}
\Phi\left(T(P T)^{m-1} p\right) & =\limsup _{n \rightarrow \infty} d\left(T(P T)^{m-1} x_{n}, T(P T)^{m-1} p\right) \\
& \leq \limsup _{n \rightarrow \infty}\left\{d\left(x_{n}, p\right)+v_{m} \zeta\left(d\left(x_{n}, p\right)\right)+\mu_{m}\right\} .
\end{aligned}
$$

Letting $m \rightarrow \infty$ and taking superior limit on both sides, we get that

$$
\limsup _{m \rightarrow \infty} \Phi\left(T(P T)^{m-1} p\right) \leq \Phi(p) .
$$

Furthermore, for any $n, m \geq 1$, it follows from inequality (1.3) with $t=\frac{1}{2}$ that

$$
\begin{aligned}
& d^{2}\left(x_{n}, \frac{p \oplus T(P T)^{m-1}(p)}{2}\right) \\
& \quad \leq \frac{1}{2} d^{2}\left(x_{n}, p\right)+\frac{1}{2} d^{2}\left(x_{n}, T(P T)^{m-1}(p)\right)-\frac{1}{4} d^{2}\left(p, T(P T)^{m-1}(p)\right) .
\end{aligned}
$$

Letting $n \rightarrow \infty$ and taking superior limit on both sides of the above inequality, for any $m \geq 1$, we get

$$
\begin{aligned}
& \Phi\left(\frac{p \oplus T(P T)^{m-1}(p)}{2}\right)^{2} \\
& \quad \leq \frac{1}{2} \Phi(p)^{2}+\frac{1}{2} \Phi\left(T(P T)^{m-1}(p)\right)^{2}-\frac{1}{4} d^{2}\left(p, T(P T)^{m-1}(p)\right)
\end{aligned}
$$

Since $A\left(\left\{x_{n}\right\}\right)=\{p\}$, for any $m \geq 1$, we have

$$
\begin{aligned}
\Phi(p)^{2} & \leq \Phi\left(\frac{p \oplus T(P T)^{m-1}(p)}{2}\right)^{2} \\
& \leq \frac{1}{2} \Phi(p)^{2}+\frac{1}{2} \Phi\left(T(P T)^{m-1}(p)\right)^{2}-\frac{1}{4} d^{2}\left(p, T(P T)^{m-1}(p)\right) .
\end{aligned}
$$

This implies that

$$
d^{2}\left(p, T(P T)^{m-1}(p)\right) \leq 2 \Phi\left(T(P T)^{m-1}(p)\right)^{2}-2 \Phi(p)^{2} .
$$


From (2.8) and (2.12), we have $\lim _{m \rightarrow \infty} d\left(p, T(P T)^{m-1} p\right)=0$. Hence we have

$$
\begin{aligned}
d(T p, p) & \leq d\left(T p, T(P T)^{m} p\right)+d\left(T(P T)^{m} p, p\right) \\
& \leq L d\left(p,(P T)^{m} p\right)+d\left(T(P T)^{m} p, p\right) \\
& =L d\left(P p,(P T)(P T)^{m-1} p\right)+d\left(T(P T)^{m} p, p\right) \\
& \leq L d\left(p, T(P T)^{m-1} p\right)+d\left(T(P T)^{m} p, p\right) \rightarrow 0 \quad(\text { as } m \rightarrow \infty),
\end{aligned}
$$

i.e., $p=T p$ as desired.

The following theorem can be obtained from Theorem 2.6 immediately which is a generalization of Kirk et al. [4, Proposition 3.7], Xu [30], Chang et al. [31] and Chidume et al. [29, Theorem 3.4].

Theorem 2.7 Let $C$ be a closed and convex subset of a complete CAT(0) space X. Let T be a mapping satisfying one of the following conditions:

(1) $T: C \rightarrow C$ is an asymptotically nonexpansive mapping with a sequence

$$
\left\{k_{n}\right\} \subset[1, \infty), k_{n} \rightarrow 1 ;
$$

(2) $T: C \rightarrow X$ is an asymptotically nonexpansive nonself mapping with a sequence $\left\{k_{n}\right\} \subset[1, \infty), k_{n} \rightarrow 1 ;$

(3) $T: C \rightarrow C$ is a $\left(\left\{v_{n}\right\},\left\{\mu_{n}\right\}, \zeta\right)$-total asymptotically nonexpansive mapping.

Let $\left\{x_{n}\right\}$ be a bounded sequence in $C$ such that $\lim _{n \rightarrow \infty} d\left(x_{n}, T x_{n}\right)=0$ and $\Delta-\lim _{n \rightarrow \infty} x_{n}=$ p. Then $T p=p$.

\section{$3 \Delta$-convergence theorems for total asymptotically nonexpansive mappings in $\mathrm{CAT}(0)$ spaces}

In this section we prove some $\Delta$-convergence theorems for the mixed Agarwal-O'ReganSahu type iterative scheme [27]

$$
\left\{\begin{array}{l}
x_{1} \in C, \\
x_{n+1}=P\left(\left(1-\alpha_{n}\right) S_{1}^{n} x_{n} \oplus \alpha_{n} T_{1}\left(P T_{1}\right)^{n-1} y_{n}\right), \quad n \geq 1, \\
y_{n}=P\left(\left(1-\beta_{n}\right) S_{2}^{n} x_{n} \oplus \beta_{n} T_{2}\left(P T_{2}\right)^{n-1} x_{n}\right),
\end{array}\right.
$$

where $C$ is a nonempty bounded closed and convex subset of a complete CAT(0) space $X, P$ is a nonexpansive retraction of $X$ onto $C, T_{i}: C \rightarrow X, i=1,2$, is a uniformly $L_{i}$-Lipschitzian and $\left(\left\{v_{n}^{(i)}\right\},\left\{\mu_{n}^{(i)}\right\}, \zeta^{(i)}\right)$-total asymptotically nonexpansive nonself mapping (defined by (1.7)), and $S_{i}: C \rightarrow C, i=1,2$, is a uniformly $\tilde{L}_{i}$-Lipschitzian and $\left(\left\{\tilde{v}_{n}^{(i)}\right\},\left\{\tilde{\mu}_{n}^{(i)}\right\}, \tilde{\zeta}^{(i)}\right)$ total asymptotically nonexpansive mapping (defined by (1.6)) such that the following conditions are satisfied:

(1) $\sum_{n=1}^{\infty} v_{n}^{(i)}<\infty, \sum_{n=1}^{\infty} \mu_{n}^{(i)}<\infty, \sum_{n=1}^{\infty} \tilde{v}_{n}^{(i)}<\infty, \sum_{n=1}^{\infty} \tilde{\mu}_{n}^{(i)}<\infty, i=1,2$;

(2) There exists a constant $M^{*}>0$ such that $\zeta^{(i)}(r) \leq M^{*} r, \tilde{\zeta}^{(i)}(r) \leq M^{*} r, \forall r \geq 0, i=1,2$.

Remark 3.1 Without loss of generality, in the sequel, we can assume that $S_{i}: C \rightarrow C$ and $T_{i}: C \rightarrow X, i=1,2$, both are uniformly $L$-Lipschitzian and $\left(\left\{v_{n}\right\},\left\{\mu_{n}\right\}, \zeta\right)$-total asymptotically nonexpansive mappings satisfying the conditions (1) and (2). In fact, letting $v_{n}=\max \left\{v_{n}^{(i)}, \tilde{v}_{n}^{(i)}, i=1,2\right\}, \mu_{n}=\max \left\{\mu_{n}^{(i)}, \tilde{\mu}_{n}^{(i)}, i=1,2\right\}, L=\max \left\{L_{i}, \tilde{L}_{i}, i=1,2\right\}$ and $\zeta=$ 
$\max \left\{\zeta^{(i)}, \tilde{\zeta}^{(i)}, i=1,2\right\}$, then $S_{i}: C \rightarrow C$ and $T_{i}: C \rightarrow X, i=1,2$, are the mappings satisfying the required conditions.

The following lemmas will be used to prove our main results.

Lemma 3.2 (Chang et al. [24]) Let $X$ be a CAT(0) space, $x \in X$ be a given point and $\left\{t_{n}\right\}$ be a sequence in $[b, c]$ with $b, c \in(0,1)$ and $0<b(1-c) \leq \frac{1}{2}$. Let $\left\{x_{n}\right\}$ and $\left\{y_{n}\right\}$ be any sequences in $X$ such that

$$
\begin{aligned}
& \limsup _{n \rightarrow \infty} d\left(x_{n}, x\right) \leq r, \quad \underset{n \rightarrow \infty}{\limsup } d\left(y_{n}, x\right) \leq r \quad \text { and } \\
& \lim _{n \rightarrow \infty} d\left(\left(1-t_{n}\right) x_{n} \oplus t_{n} y_{n}, x\right)=r,
\end{aligned}
$$

for some $r \geq 0$. Then

$$
\lim _{n \rightarrow \infty} d\left(x_{n}, y_{n}\right)=0
$$

Lemma 3.3 Let $\left\{a_{n}\right\},\left\{\lambda_{n}\right\}$ and $\left\{c_{n}\right\}$ be the sequences of nonnegative numbers such that

$$
a_{n+1} \leq\left(1+\lambda_{n}\right) a_{n}+c_{n}, \quad \forall n \geq 1
$$

If $\sum_{n=1}^{\infty} \lambda_{n}<\infty$ and $\sum_{n=1}^{\infty} c_{n}<\infty$, then $\lim _{n \rightarrow \infty} a_{n}$ exists. If there exists a subsequence $\left\{a_{n_{i}}\right\} \subset\left\{a_{n}\right\}$ such that $a_{n_{i}} \rightarrow 0$, then $\lim _{n \rightarrow \infty} a_{n}=0$.

Lemma 3.4 [2] Let $X$ be a complete CAT(0) space, $\left\{x_{n}\right\}$ be a bounded sequence in $X$ with $A\left(\left\{x_{n}\right\}\right)=\{p\}$, and $\left\{u_{n}\right\}$ be a subsequence of $\left\{x_{n}\right\}$ with $A\left(\left\{u_{n}\right\}\right)=\{u\}$ and the sequence $\left\{d\left(x_{n}, u\right)\right\}$ converges, then $p=u$.

Now we are in a position to give the main results of this paper.

Theorem 3.5 Let $C$ be a bounded closed and convex subset of a complete CAT(0)X. Let $T_{i}: C \rightarrow X, i=1,2$, be a uniformly L-Lipschitzian and $\left(\left\{v_{n}\right\},\left\{\mu_{n}\right\}, \zeta\right)$-total asymptotically nonexpansive nonself mapping, and let $S_{i}: C \rightarrow C, i=1,2$, be a uniformly L-Lipschitzian and $\left(\left\{v_{n}\right\},\left\{\mu_{n}\right\}, \zeta\right)$-total asymptotically nonexpansive mapping. If $\mathcal{F}:=\bigcap_{i=1}^{2} F\left(T_{i}\right) \cap F\left(S_{i}\right) \neq$ $\emptyset$ and the following conditions are satisfied:

(i) $\sum_{n=1}^{\infty} v_{n}<\infty ; \sum_{n=1}^{\infty} \mu_{n}<\infty$;

(ii) there exist constants $a, b \in(0,1)$ with $0<b(1-c) \leq \frac{1}{2}$ such that $\left\{\alpha_{n}\right\} \subset[a, b]$;

(iii) there exists a constant $M^{*}>0$ such that $\zeta(r) \leq M^{*} r, r \geq 0$;

(iv) $d\left(x, T_{i} y\right) \leq d\left(S_{i} x, T_{i} y\right)$ for all $x, y \in C$ and $i=1,2$,

then the sequence $\left\{x_{n}\right\}$ defined by (3.1) $\Delta$-converges to some point $p^{*} \in \mathcal{F}$ (a common fixed point of $T_{i}$ and $\left.S_{i}, i=1,2\right)$.

Proof (I) First we prove that the following limits exist

$$
\lim _{n \rightarrow \infty} d\left(x_{n}, p\right) \quad \text { for each } p \in \mathcal{F} \quad \text { and } \quad \lim _{n \rightarrow \infty} d\left(x_{n}, \mathcal{F}\right)
$$


In fact, since $p \in \mathcal{F}, p=P p$. In addition, since $S_{i}$ and $T_{i}, i=1,2$, are total asymptotically nonexpansive mappings, by the condition (iii), we have

$$
\begin{aligned}
d\left(y_{n}, p\right) & =d\left(P\left(\left(1-\beta_{n}\right) S_{2}^{n} x_{n} \oplus \beta_{n} T_{2}\left(P T_{2}\right)^{n-1} x_{n}\right), P p\right) \\
& \leq d\left(\left(1-\beta_{n}\right) S_{2}^{n} x_{n} \oplus \beta_{n} T_{2}\left(P T_{2}\right)^{n-1} x_{n}, p\right) \\
& \leq\left(1-\beta_{n}\right) d\left(S_{2}^{n} x_{n}, p\right)+\beta_{n} d\left(T_{2}\left(P T_{2}\right)^{n-1} x_{n}, p\right) \\
& =\left(1-\beta_{n}\right)\left\{d\left(x_{n}, p\right)+v_{n} \zeta\left(d\left(x_{n}, p\right)\right)+\mu_{n}\right\}+\beta_{n}\left\{d\left(x_{n}, p\right)+v_{n} \zeta\left(d\left(x_{n}, p\right)\right)+\mu_{n}\right\} \\
& =d\left(x_{n}, p\right)+v_{n} \zeta\left(d\left(x_{n}, p\right)\right)+\mu_{n} \\
& \leq\left(1+v_{n} M^{*}\right) d\left(x_{n}, p\right)+\mu_{n}
\end{aligned}
$$

and

$$
\begin{aligned}
d\left(x_{n+1}, p\right) & =d\left(P\left(\left(1-\alpha_{n}\right) S_{1}^{n} x_{n} \oplus \alpha_{n} T_{1}\left(P T_{1}\right)^{n-1} y_{n}\right), P p\right) \\
& \leq d\left(\left(1-\alpha_{n}\right) S_{1}^{n} x_{n} \oplus \alpha_{n} T_{1}\left(P T_{1}\right)^{n-1} y_{n}, p\right) \\
& \leq\left(1-\alpha_{n}\right) d\left(S_{1}^{n} x_{n}, p\right)+\alpha_{n} d\left(T_{1}\left(P T_{1}\right)^{n-1} y_{n}, p\right) \\
& =\left(1-\alpha_{n}\right)\left\{d\left(x_{n}, p\right)+v_{n} \zeta\left(d\left(x_{n}, p\right)\right)+\mu_{n}\right\}+\alpha_{n}\left\{d\left(y_{n}, p\right)+v_{n} \zeta\left(d\left(y_{n}, p\right)\right)+\mu_{n}\right\} \\
& \leq\left(1-\alpha_{n}\right)\left\{\left(1+v_{n} M^{*}\right) d\left(x_{n}, p\right)+\mu_{n}\right\}+\alpha_{n}\left\{\left(1+v_{n} M^{*}\right) d\left(y_{n}, p\right)+\mu_{n}\right\} .
\end{aligned}
$$

Substituting (3.4) into (3.5) and simplifying it, we have

$$
d\left(x_{n+1}, p\right) \leq\left(1+\sigma_{n}\right) d\left(x_{n}, p\right)+\xi_{n}, \quad \forall n \geq 1 \text { and } p \in \mathcal{F},
$$

and so

$$
d\left(x_{n+1}, \mathcal{F}\right) \leq\left(1+\sigma_{n}\right) d\left(x_{n}, \mathcal{F}\right)+\xi_{n}, \quad \forall n \geq 1,
$$

where $\sigma_{n}=v_{n} M^{*}\left(1+\alpha_{n}\left(1+v_{n} M^{*}\right)\right), \xi_{n}=\left(1+\alpha_{n}\left(1+v_{n} M^{*}\right)\right) \mu_{n}$. By virtue of the condition (i),

$$
\sum_{n=1}^{\infty} \sigma_{n}<\infty \text { and } \sum_{n=1}^{\infty} \xi_{n}<\infty
$$

By Lemma 3.3 the $\operatorname{limits}_{\lim _{n \rightarrow \infty}} d\left(x_{n}, \mathcal{F}\right)$ and $\lim _{n \rightarrow \infty} d\left(x_{n}, p\right)$ exist for each $p \in \mathcal{F}$.

(II) Next we prove that

$$
\lim _{n \rightarrow \infty} d\left(x_{n}, T_{i} x_{n}\right)=0, \quad \lim _{n \rightarrow \infty} d\left(x_{n}, S_{i} x_{n}\right)=0, \quad i=1,2 .
$$

In fact, it follows from (3.3) that for each given $p \in \mathcal{F}, \lim _{n \rightarrow \infty} d\left(x_{n}, p\right)$ exists. Without loss of generality, we can assume that

$$
\lim _{n \rightarrow \infty} d\left(x_{n}, p\right)=r \geq 0 .
$$

From (3.4) we have

$$
\liminf _{n \rightarrow \infty} d\left(y_{n}, p\right) \leq \limsup _{n \rightarrow \infty} d\left(y_{n}, p\right) \leq \lim _{n \rightarrow \infty}\left\{\left(1+v_{n} M^{*}\right) d\left(x_{n}, p\right)+\mu_{n}\right\}=r .
$$


Since

$$
\begin{aligned}
d\left(T_{1}\left(P T_{1}\right)^{n-1} y_{n}, p\right) & =d\left(T_{1}\left(P T_{1}\right)^{n-1} y_{n}, T_{1}\left(P T_{1}\right)^{n-1} p\right) \leq d\left(y_{n}, p\right)+v_{n} \zeta\left(d\left(y_{n}, p\right)\right)+\mu_{n} \\
& \leq\left(1+v_{n} M^{*}\right) d\left(y_{n}, p\right)+\mu_{n}, \quad \forall n \geq 1,
\end{aligned}
$$

and

$$
d\left(S_{1}^{n} x_{n}, p\right) \leq d\left(x_{n}, p\right)+v_{n} \zeta\left(d\left(x_{n}, p\right)\right)+\mu_{n} \leq\left(1+v_{n} M^{*}\right) d\left(x_{n}, p\right)+\mu_{n}, \quad \forall n \geq 1,
$$

then we have

$$
\limsup _{n \rightarrow \infty} d\left(T_{1}\left(P T_{1}\right)^{n-1} y_{n}, p\right) \leq r
$$

and

$$
\limsup _{n \rightarrow \infty} d\left(S_{1}^{n} x_{n}, p\right) \leq r
$$

In addition, it follows from (3.6) that

$$
d\left(x_{n+1}, p\right) \leq d\left(\left(1-\alpha_{n}\right) S_{1}^{n} x_{n} \oplus \alpha_{n} T_{1}\left(P T_{1}\right)^{n-1} y_{n}, p\right) \leq\left(1+\sigma_{n}\right) d\left(x_{n}, p\right)+\xi_{n} .
$$

This implies that

$$
\lim _{n \rightarrow \infty} d\left(\left(1-\alpha_{n}\right) S_{1}^{n} x_{n} \oplus \alpha_{n} T_{1}\left(P T_{1}\right)^{n-1} y_{n}, p\right)=r
$$

From (3.12)-(3.14) and Lemma 3.2, one gets that

$$
\lim _{n \rightarrow \infty} d\left(S_{1}^{n} x_{n}, T_{1}\left(P T_{1}\right)^{n-1} y_{n}\right)=0
$$

By the same method, we can also prove that

$$
\lim _{n \rightarrow \infty} d\left(S_{2}^{n} x_{n}, T_{2}\left(P T_{2}\right)^{n-1} x_{n}\right)=0
$$

By virtue of the condition (iv), it follows from (3.15) and (3.16) that

$$
\lim _{n \rightarrow \infty} d\left(x_{n}, T_{1}\left(P T_{1}\right)^{n-1} y_{n}\right) \leq \lim _{n \rightarrow \infty} d\left(S_{1}^{n} x_{n}, T_{1}\left(P T_{1}\right)^{n-1} y_{n}\right)=0
$$

and

$$
\lim _{n \rightarrow \infty} d\left(x_{n}, T_{2}\left(P T_{2}\right)^{n-1} x_{n}\right) \leq \lim _{n \rightarrow \infty} d\left(S_{2}^{n} x_{n}, T_{2}\left(P T_{2}\right)^{n-1} x_{n}\right)=0 .
$$

Since $S_{2}^{n} x_{n} \in C, S_{2}^{n} x_{n}=P S_{2}^{n} x_{n}$. By (3.1) and (3.16) we have

$$
\begin{aligned}
d\left(y_{n}, S_{2}^{n} x_{n}\right) & \leq d\left(\left(1-\beta_{n}\right) S_{2}^{n} x_{n} \oplus \beta_{n} T_{2}\left(P T_{2}\right)^{n-1} x_{n}, S_{2}^{n} x_{n}\right) \\
& \leq \beta_{n} d\left(T_{2}\left(P T_{2}\right)^{n-1} x_{n}, S_{2}^{n} x_{n}\right) \rightarrow 0 \quad(\operatorname{as} n \rightarrow \infty)
\end{aligned}
$$


Observe that

$$
d\left(x_{n}, y_{n}\right) \leq d\left(x_{n}, T_{2}\left(P T_{2}\right)^{n-1} x_{n}\right)+d\left(T_{2}\left(P T_{2}\right)^{n-1} x_{n}, S_{2}^{n} x_{n}\right)+d\left(S_{2}^{n} x_{n}, y_{n}\right) .
$$

From (3.18) and (3.19) we get

$$
\lim _{n \rightarrow \infty} d\left(x_{n}, y_{n}\right)=0
$$

This together with (3.17) implies that

$$
\begin{aligned}
d\left(x_{n}, T_{1}\left(P T_{1}\right)^{n-1} x_{n}\right) & \leq d\left(x_{n}, T_{1}\left(P T_{1}\right)^{n-1} y_{n}\right)+d\left(T_{1}\left(P T_{1}\right)^{n-1} y_{n}, T_{1}\left(P T_{1}\right)^{n-1} x_{n}\right) \\
& =d\left(x_{n}, T_{1}\left(P T_{1}\right)^{n-1} y_{n}\right)+d\left(x_{n}, y_{n}\right)+v_{n} \zeta\left(d\left(x_{n}, y_{n}\right)\right)+\mu_{n} \\
& \leq d\left(x_{n}, T_{1}\left(P T_{1}\right)^{n-1} y_{n}\right)+\left(1+v_{n} M^{*}\right) d\left(x_{n}, y_{n}\right)+\mu_{n} \rightarrow 0 .
\end{aligned}
$$

On the other hand, by the condition (iv), $d\left(x_{n}, T_{1}\left(P T_{1}\right)^{n-1} x_{n}\right) \leq d\left(S_{1}^{n} x_{n}, T_{1}\left(P T_{1}\right)^{n-1} x_{n}\right)$. Hence from (3.17) and (3.20), we have

$$
\begin{aligned}
& d\left(S_{1}^{n} x_{n}, T_{1}\left(P T_{1}\right)^{n-1} x_{n}\right) \\
& \quad \leq d\left(S_{1}^{n} x_{n}, T_{1}\left(P T_{1}\right)^{n-1} y_{n}\right)+d\left(T_{1}\left(P T_{1}\right)^{n-1} y_{n}, T_{1}\left(P T_{1}\right)^{n-1} x_{n}\right) \\
& \quad \leq d\left(S_{1}^{n} x_{n}, T_{1}\left(P T_{1}\right)^{n-1} y_{n}\right)+L d\left(y_{n}, x_{n}\right) \rightarrow 0 \quad(\text { as } n \rightarrow \infty) .
\end{aligned}
$$

By the condition (iv), $d\left(x_{n}, T_{1}\left(P T_{1}\right)^{n-1} x_{n}\right) \leq d\left(S_{1}^{n} x_{n}, T_{1}\left(P T_{1}\right)^{n-1} x_{n}\right)$. Hence from (3.22) we have that

$$
d\left(S_{1}^{n} x_{n}, x_{n}\right) \leq d\left(S_{1}^{n} x_{n}, T_{1}\left(P T_{1}\right)^{n-1} x_{n}\right)+d\left(T_{1}\left(P T_{1}\right)^{n-1} x_{n}, x_{n}\right) \rightarrow 0 \quad(\text { as } n \rightarrow \infty) .
$$

This together with (3.17) shows that

$$
\begin{aligned}
d\left(x_{n+1}, x_{n}\right) \leq & d\left(\left(1-\alpha_{n}\right) S_{1}^{n} x_{n} \oplus \alpha_{n} T_{1}\left(P T_{1}\right)^{n-1} y_{n}, x_{n}\right) \\
\leq & \left(1-\alpha_{n}\right) d\left(S_{1}^{n} x_{n}, x_{n}\right)+\alpha_{n} d\left(T_{1}\left(P T_{1}\right)^{n-1} y_{n}, x_{n}\right) \rightarrow 0 \\
& (\text { as } n \rightarrow \infty) .
\end{aligned}
$$

Hence from (3.18), (3.21) and (3.23), for each $i=1,2$, we have

$$
\begin{aligned}
d\left(x_{n}, T_{i} x_{n}\right) \leq & d\left(x_{n}, x_{n+1}\right)+d\left(x_{n+1}, T_{i}\left(P T_{i}\right)^{n} x_{n+1}\right) \\
& +d\left(T_{i}\left(P T_{i}\right)^{n} x_{n+1}, T_{i}\left(P T_{i}\right)^{n} x_{n}\right)+d\left(T_{i}\left(P T_{i}\right)^{n} x_{n}, T_{i} x_{n}\right) \\
\leq & (1+L) d\left(x_{n}, x_{n+1}\right)+d\left(x_{n+1}, T_{i}\left(P T_{i}\right)^{n} x_{n+1}\right)+L d\left(\left(P T_{i}\right)^{n} x_{n}, x_{n}\right) \\
= & (1+L) d\left(x_{n}, x_{n+1}\right)+d\left(x_{n+1}, T_{i}\left(P T_{i}\right)^{n} x_{n+1}\right)+L d\left(P T_{i}\left(P T_{i}\right)^{n-1} x_{n}, P x_{n}\right) \\
\leq & (1+L) d\left(x_{n}, x_{n+1}\right)+d\left(x_{n+1}, T_{i}\left(P T_{i}\right)^{n} x_{n+1}\right) \\
& +L d\left(T_{i}\left(P T_{i}\right)^{n-1} x_{n}, x_{n}\right) \rightarrow 0 .
\end{aligned}
$$


By virtue of the condition (iv), $d\left(S_{i} x_{n}, T_{i}\left(P T_{i}\right)^{n-1} x_{n}\right) \leq d\left(S_{i}^{n} x_{n}, T_{i}\left(P T_{i}\right)^{n-1} x_{n}\right)$. It follows from (3.18), (3.21) and (3.22) that

$$
\begin{aligned}
d\left(x_{n}, S_{i} x_{n}\right) & \leq d\left(x_{n}, T_{i}\left(P T_{i}\right)^{n-1} x_{n}\right)+d\left(S_{i} x_{n}, T_{i}\left(P T_{i}\right)^{n-1} x_{n}\right) \\
& \leq d\left(x_{n}, T_{i}\left(P T_{i}\right)^{n-1} x_{n}\right)+d\left(S_{i}^{n} x_{n}, T_{i}\left(P T_{i}\right)^{n-1} x_{n}\right) \rightarrow 0 \quad(\text { as } n \rightarrow \infty) .
\end{aligned}
$$

Equation (3.9) is proved.

(III) Now we prove that

$$
\omega_{w}\left(x_{n}\right):=\bigcup_{\left\{u_{n}\right\} \subset\left\{x_{n}\right\}} A\left(\left\{u_{n}\right\}\right) \subset \mathcal{F}
$$

and $\omega_{w}\left(x_{n}\right)$ consists of exactly one point.

In fact, let $u \in \omega_{w}\left(x_{n}\right)$, then there exists a subsequence $\left\{u_{n}\right\}$ of $\left\{x_{n}\right\}$ such that $A\left(\left\{u_{n}\right\}\right)=$ $\{u\}$. By Lemma 2.3, there exists a subsequence $\left\{v_{n}\right\}$ of $\left\{u_{n}\right\}$ such that $\Delta-\lim _{n \rightarrow \infty} v_{n}=v \in C$. In view of (3.9), $\lim _{n \rightarrow \infty} d\left(v_{n}, T_{i} v_{n}\right)=0, \lim _{n \rightarrow \infty} d\left(v_{n}, S_{i} v_{n}\right)=0, i=1,2$. It follows from Theorem 2.7 that $v \in \mathcal{F}$. So, by (3.3), the $\operatorname{limit}_{n \rightarrow \infty} d\left(x_{n}, v\right)$ exists. By Lemma $3.4 u=v$. This implies that $\omega_{w}\left(x_{n}\right) \subset \mathcal{F}$.

Next we prove that $\omega_{w}\left(x_{n}\right)$ consists of exactly one point. Let $\left\{u_{n}\right\}$ be a subsequence of $\left\{x_{n}\right\}$ with $A\left(\left\{u_{n}\right\}\right)=\{u\}$ and let $A\left(\left\{x_{n}\right\}\right)=\{x\}$. Since $u \in \omega_{w}\left(x_{n}\right) \subset \mathcal{F}$, from (3.3) the limit $\lim _{n \rightarrow \infty} d\left(x_{n}, u\right)$ exists. In view of Lemma 3.4, $x=u$. The conclusion is proved.

(IV) Finally we prove $\left\{x_{n}\right\} \Delta$-converges to a point in $\mathcal{F}$.

In fact, it follows from (3.3) that $\left\{d\left(x_{n}, p\right)\right\}$ is convergent for each $p \in \mathcal{F}$. By (3.9) and (3.26), $\lim _{n \rightarrow \infty} d\left(x_{n}, S_{i} x_{n}\right)=0, \lim _{n \rightarrow \infty} d\left(x_{n}, T_{i} x_{n}\right)=0, \omega_{w}\left(x_{n}\right) \subset \mathcal{F}$ and $\omega_{w}\left(x_{n}\right)$ consists of exactly one point. This shows that $\left\{x_{n}\right\} \Delta$-converges to a point of $\mathcal{F}$.

The conclusion of Theorem 3.5 is proved.

Remark 3.6 (1) Now we give an example which satisfies the condition (iv) in Theorem 3.5. Let $C=[-1,1]$ be a subset in $\mathcal{R}$. Define two mappings $S_{1}=S_{2}=S, T_{1}=T_{2}=T: C \rightarrow C$ by

$$
T(x)= \begin{cases}-2 \sin \frac{x}{2}, & \text { if } x \in[0,1], \\ 2 \sin \frac{x}{2}, & \text { if } x \in[-1,0)\end{cases}
$$

and

$$
S(x)= \begin{cases}x, & \text { if } x \in[0,1], \\ -x, & \text { if } x \in[-1,0)\end{cases}
$$

It is proved in Guo [28] that both $S$ and $T$ are asymptotically nonexpansive mappings (therefore they are total asymptotically nonexpansive mappings) with $F(T) \cap F(S) \neq \emptyset$ and satisfy the condition (iv).

(2) Theorem 3.5 contains the main results of Sahin [26], Khan Abbas [22], Khan et al. [23] and Chang et al. [24] as its special cases. Theorem 3.5 also extends the main result of Guo et al. [28] from a Banach space to a CAT(0) space. 
The following results can be obtained from Theorem 3.5 immediately.

Theorem 3.7 Let $C, X$ and $T_{i}: C \rightarrow X, i=1,2$ be the same as in Theorem 3.5. If $\mathcal{F}:=$ $\bigcap_{i=1}^{2} F\left(T_{i}\right) \neq \varnothing$ and the following conditions are satisfied:

(i) $\sum_{n=1}^{\infty} v_{n}<\infty ; \sum_{n=1}^{\infty} \mu_{n}<\infty$;

(ii) there exist constants $a, b \in(0,1)$ with $0<b(1-c) \leq \frac{1}{2}$ such that $\left\{\alpha_{n}\right\} \subset[a, b]$.

(iii) there exists a constant $M^{*}>0$ such that $\zeta(r) \leq M^{*} r, r \geq 0$;

then the sequence $\left\{x_{n}\right\}$ defined by

$$
\left\{\begin{array}{l}
x_{1} \in C \\
x_{n+1}=P\left(\left(1-\alpha_{n}\right) x_{n} \oplus \alpha_{n} T_{1}\left(P T_{1}\right)^{n-1} y_{n}\right), \quad n \geq 1, \\
y_{n}=P\left(\left(1-\beta_{n}\right) x_{n} \oplus \beta_{n} T_{2}\left(P T_{2}\right)^{n-1} x_{n}\right),
\end{array}\right.
$$

$\Delta$-converges to a common fixed point of $T_{1}$ and $T_{2}$.

Proof Take $S_{i}=I$ (the identity mapping on $C$ ) in Theorem 3.5 and note that in this case the condition (iv) in Theorem 3.5 is satisfied automatically. Hence the conclusion of Theorem 3.7 can be obtained from Theorem 3.5 immediately.

Theorem 3.8 Let $C$ and $X$ be the same as in Theorem 3.5. Let $T_{i}: C \rightarrow C$ and $S_{i}: C \rightarrow C$, $i=1,2$, be uniformly L-Lipschitzian and $\left(\left\{v_{n}\right\},\left\{\mu_{n}\right\}, \zeta\right)$-total asymptotically nonexpansive mappings. If $\mathcal{F}:=\bigcap_{i=1}^{2} F\left(T_{i}\right) \cap F\left(S_{i}\right) \neq \emptyset$ and the (i)-(iv) in Theorem 3.5 are satisfied, then the sequence $\left\{x_{n}\right\}$ defined by

$$
\left\{\begin{array}{l}
x_{1} \in C, \\
x_{n+1}=\left(1-\alpha_{n}\right) S_{1}^{n} x_{n} \oplus \alpha_{n} T_{1}^{n} y_{n}, \quad n \geq 1, \\
y_{n}=\left(1-\beta_{n}\right) S_{2}^{n} x_{n} \oplus \beta_{n} T_{2}^{n} x_{n},
\end{array}\right.
$$

$\Delta$-converges to a common fixed point of $T_{i}$ and $S_{i}, i=1,2$.

Proof Since $T_{i}, i=1,2$, is a self-mapping from $C$ to $C$, take $P=I$ (the identity mapping on $C$ ), then $T_{i}\left(P T_{i}\right)^{n-1}=T_{i}^{n}$. The conclusion of Theorem 3.8 is obtained from Theorem 3.5.

Remark 3.9 Theorem 3.8 improves and extends the main results of Agawal O'Regan Sahu [27] from a Banach space to a CAT(0) space. As well as it also extends and improves the main results in Sahin [26].

\section{Strong convergence theorems for total asymptotically nonexpansive mappings in $\mathrm{CAT}(0)$ spaces}

Recall that a mapping $T: C \rightarrow X$ is said to be demi-compact if for any sequence $\left\{x_{n}\right\}$ in $C$ such that $d\left(x_{n}, T x_{n}\right) \rightarrow 0$ (as $n \rightarrow \infty$ ), there exists a subsequence $\left\{x_{n_{i}}\right\} \subset\left\{x_{n}\right\}$ such that $\left\{x_{n_{i}}\right\}$ converges strongly (i.e., in metric topology) to some point $x^{*} \in C$.

Theorem 4.1 Under the assumptions of Theorem 3.5, if one of $S_{1}, S_{2}, T_{1}$ and $T_{2}$ is demicompact, then the sequence defined by (3.1) converges strongly (i.e., in metric topology) to a common fixed point $p \in \mathcal{F}$. 
Proof By virtue of (3.9): $\lim _{n \rightarrow \infty} d\left(x_{n}, T_{i} x_{n}\right)=0, \lim _{n \rightarrow \infty} d\left(x_{n}, S_{i} x_{n}\right)=0, i=1,2$ and one of $S_{1}, S_{2}, T_{1}$ and $T_{2}$ is demi-compact, there exists a subsequence $\left\{x_{n_{i}}\right\} \subset\left\{x_{n}\right\}$ such that $\left\{x_{n_{i}}\right\}$ converges strongly to some point $p \in C$. Moreover, by the continuity of $S_{1}, S_{2}, T_{1}$ and $T_{2}$, for each $i=1,2$, we have

$$
\begin{aligned}
& d\left(p, S_{i} p\right)=\lim _{n \rightarrow \infty} d\left(x_{n_{i}}, S_{i} x_{n_{i}}\right)=0, \\
& d\left(p, T_{i} p\right)=\lim _{n \rightarrow \infty} d\left(x_{n_{i}}, T_{i} x_{n_{i}}\right)=0 .
\end{aligned}
$$

This implies that $p \in \mathcal{F}$. Again by (3.3) the $\operatorname{limit}_{n \rightarrow \infty} d\left(x_{n}, p\right)$ exists. Hence we have $\lim _{n \rightarrow \infty} d\left(x_{n}, p\right)=0$. This completes the proof of Theorem 4.1.

Theorem 4.2 Under the assumptions of Theorem 3.5, if there exists a nondecreasing function $f:[0, \infty) \rightarrow[0, \infty)$ with $f(0)=0, f(r)>0, \forall r>0$ such that

$$
f(d(x, \mathcal{F})) \leq d\left(x, S_{1} x\right)+d\left(x, S_{2} x\right)+d\left(x, T_{1} x\right)+d\left(x, T_{2} x\right), \quad \forall x \in C,
$$

then the sequence $\left\{x_{n}\right\}$ defined by (3.1) converges strongly (i.e., in metric topology) to a common fixed point $p^{*} \in \mathcal{F}$.

Proof It follows from (3.9) that

$$
\lim _{n \rightarrow \infty} d\left(x_{n}, T_{i} x_{n}\right)=0, \quad \lim _{n \rightarrow \infty} d\left(x_{n}, S_{i} x_{n}\right)=0, \quad i=1,2 .
$$

Therefore we have $\lim _{n \rightarrow \infty} f\left(d\left(x_{n}, \mathcal{F}\right)\right)=0$. Since $f$ is a nondecreasing function with $f(0)=0$ and $f(r)>0, r>0$, we have $\lim _{n \rightarrow \infty} d\left(x_{n}, \mathcal{F}\right)=0$. Next we prove that $\left\{x_{n}\right\}$ is a Cauchy sequence in $C$. In fact, it follows from (3.6) that for any $p \in \mathcal{F}$

$$
d\left(x_{n+1}, p\right) \leq\left(1+\sigma_{n}\right) d\left(x_{n}, p\right)+\xi_{n}, \quad \forall n \geq 1,
$$

where $\sum_{n=1}^{\infty} \sigma_{n}<\infty$ and $\sum_{n=1}^{\infty} \xi_{n}<\infty$. Hence for any positive integers $n, m$, we have

$$
\begin{aligned}
d\left(x_{n+m}, x_{n}\right) & \leq d\left(x_{n+m}, p\right)+d\left(x_{n}, p\right) \\
& \leq\left(1+\sigma_{n+m-1}\right) d\left(x_{n+m-1}, p\right)+\xi_{n+m-1}+d\left(x_{n}, p\right) .
\end{aligned}
$$

Since for each $x \geq 0,1+x \leq e^{x}$, we have

$$
\begin{aligned}
d\left(x_{n+m}, x_{n}\right) \leq & e^{\sigma_{n+m-1}} d\left(x_{n+m-1}, p\right)+\xi_{n+m-1}+d\left(x_{n}, p\right) \\
\leq & e^{\sigma_{n+m-1}+\sigma_{n+m-2}} d\left(x_{n+m-2}, p\right)+e^{\sigma_{n+m-1}} \xi_{n+m-2}+\xi_{n+m-1}+d\left(x_{n}, p\right) \\
\leq & \cdots \\
\leq & e^{\sum_{i=n}^{n+m-1} \sigma_{i}} d\left(x_{n}, p\right)+e^{\sum_{i=n+1}^{n+m-1} \sigma_{i}} \xi_{n}+e^{\sum_{i=n+2}^{n+m-2} \sigma_{i}} \xi_{n+1}+\cdots \\
& +e^{\sigma_{n+m-1}} \xi_{n+m-2}+\xi_{n+m-1}+d\left(x_{n}, p\right) \\
\leq & (1+M) d\left(x_{n}, p\right)+M \sum_{i=n}^{n+m-1} \xi_{i},
\end{aligned}
$$


where $M=e^{\sum_{i=1}^{\infty} \sigma_{i}}<\infty$. By (3.3) $\lim _{n \rightarrow \infty} d\left(x_{n}, \mathcal{F}\right)=0$. Therefore we have

$$
d\left(x_{n+m}, x_{n}\right) \leq(1+M) d\left(x_{n}, \mathcal{F}\right)+M \sum_{i=n}^{n+m-1} \xi_{i} \rightarrow 0 \quad(\text { as } n, m \rightarrow \infty)
$$

This shows that $\left\{x_{n}\right\}$ is a Cauchy sequence in $C$. Since $C$ is a closed subset in a complete $\operatorname{CAT}(0)$ space $X$, it is complete. Without loss of generality, we can assume that $\left\{x_{n}\right\}$ converges strongly (i.e., in metric topology in $X$ ) to some point $p^{*} \in C$. It is easy to prove that $F\left(T_{i}\right)$ and $F\left(S_{i}\right), i=1,2$ are closed subsets in $C$, so is $\mathcal{F}$. Since $\lim _{n \rightarrow \infty} d\left(x_{n}, \mathcal{F}\right)=0, p^{*} \in \mathcal{F}$. This completes the proof of Theorem 4.2.

\section{Competing interests}

The authors declare that they have no competing interests.

\section{Authors' contributions}

All authors read and approved the final manuscript.

\section{Author details}

${ }^{1}$ College of Statistics and Mathematics, Yunnan University of Finance and Economics, Kunming, Yunnan 650221, China.

${ }^{2}$ Department of Applied Mathematics, The Hong Kong Polytechnic University, Hong Kong, China.

\section{Acknowledgements}

The authors would like to express their thanks to the referees for their helpful comments and suggestions. This work was supported by the Natural Science Foundation of Yunnan Province, Grant No. 2011 FB074.

\section{Received: 18 February 2013 Accepted: 24 April 2013 Published: 8 May 2013}

\section{References}

1. Bridson, MR, Haefliger, A: Metric Spaces of Non-Positive Curvature. Grundlehren der Mathematischen Wissenschaften, vol. 319. Springer, Berlin (1999)

2. Dhompongsa, S, Panyanak, B: On $\Delta$-convergence theorems in CAT(0) spaces. Comput. Math. Appl. 56(10), 2572-2579 (2008)

3. Lim, TC: Remarks on some fixed point theorems. Proc. Am. Math. Soc. 60, 179-182 (1976)

4. Kirk, WA, Panyanak, B: A concept of convergence in geodesic spaces. Nonlinear Anal., Theory Methods Appl. 68(12), 3689-3696 (2008)

5. Kirk, WA: Geodesic geometry and fixed point theory. In: Seminar of Mathematical Analysis (Malaga/Seville, 2002/2003). Colecc. Abierta, vol. 64, pp. 195-225. Univ. Sevilla Secr. Publ., Seville (2003)

6. Kirk, WA: Geodesic geometry and fixed point theory II. In: International Conference on Fixed Point Theo. Appl., pp. 113-142. Yokohama Publ., Yokohama (2004)

7. Dhompongsa, S, Kirk, WA, Sims, B: Fixed points of uniformly Lipschitzian mappings. Nonlinear Anal., Theory Methods Appl. 65(4), 762-772 (2006)

8. Dhompongsa, S, Kirk, WA, Panyanak, B: Nonexpansive set-valued mappings in metric and Banach spaces. J. Nonlinear Convex Anal. 8(1), 35-45 (2007)

9. Dhompongsa, S, Fupinwong, W, Kaewkhao, A: Common fixed points of a nonexpansive semigroup and a convergence theorem for Mann iterations in geodesic metric spaces. Nonlinear Anal., Theory Methods Appl. 70(12), 4268-4273 (2009)

10. Dhompongsa, S, Kaewkhao, A, Panyanak, B: On Kirk's strong convergence theorem for multivalued nonexpansive mappings on CAT(0) spaces. Nonlinear Anal. 75, 459-468 (2012)

11. Espinola, R, Fernandez-Leon, A: CAT(k)-spaces, weak convergence and fixed points. J. Math. Anal. Appl. 353(1) 410-427 (2009)

12. Laowang, W, Panyanak, B: Strong and $\Delta$-convergence theorems for multivalued mappings in CAT(0) spaces. J. Inequal. Appl. 2009, Article ID 730132 (2009)

13. Shahzad, N: Invariant approximations in CAT(0) spaces. Nonlinear Anal., Theory Methods Appl. 70(12), 4338-4340 (2009)

14. Nanjaras, B, Panyanak, B, Phuengrattana, W: Fixed point theorems and convergence theorems for Suzuki-generalized nonexpansive mappings in CAT(0) spaces. Nonlinear Anal. Hybrid Syst. 4(1), 25-31 (2010)

15. Laowang, W, Panyanak, B: Approximating fixed points of nonexpansive nonself mappings in CAT(0) spaces. Fixed Point Theory Appl. 2010, Article ID 367274 (2010). doi:10.1155/2010/367274

16. Leustean, L: A quadratic rate of asymptotic regularity for CAT(0)-spaces. J. Math. Anal. Appl. 325(1), 386-399 (2007)

17. Saejung, S: Halpern's iteration in CAT(0) spaces. Fixed Point Theory Appl. 2010, Article ID 471781 (2010). doi:10.1155/2010/471781

18. Cho, YJ, Ciric, L, Wang, SH: Convergence theorems for nonexpansive semigroups in CAT(0) spaces. Nonlinear Anal. (2011). doi:10.1016/j.na.2011.05.082

19. Abkar, A, Eslamian, M: Fixed point and convergence theorems for different classes of generalized nonexpansive mappings in CAT(0) spaces. Comput. Math. Appl. (2011). doi:10.1016/j.camwa.2011.12.075 
20. Nanjaras, B, Panyanak, B: Demiclosed principle for asymptotically nonexpansive mappings in CAT(0) spaces. Fixed Point Theory Appl. 2010, Article ID 268780 (2010). doi:10.1155/2010/268780

21. He, JS, Fang, DH, Lopez, G, Li, C: Mann's algorithm for nonexpansive mappings in CAT(0) spaces. Nonlinear Anal. 75, 459-468 (2012)

22. Khan, SH, Abbas, M: Strong and $\Delta$-convergence of some iterative schemes in CAT(0) spaces. Comput. Math. Appl. 61, 109-116 (2011)

23. Khan, AR, Khamsi, MA, Fukharuddin, $\mathrm{H}$ : Strong convergence of a general iteration scheme in CAT(0) spaces. Nonlinear Anal. 74, 783-791 (2011)

24. Chang, SS, Wang, L, Joseph Lee, HW, Chan, CK, Yang, L: Demiclosed principle and $\Delta$-convergence theorems for total asymptotically nonexpansive mappings in CAT(0) spaces. Appl. Math. Comput. 219, 2611-2617 (2012)

25. Tang, JF, Chang, SS, Joseph Lee, HW, Chan, CK: Iterative algorithm and $\Delta$-convergence theorems for total asymptotically nonexpansive mappings in CAT(0) spaces. Abstr. Appl. Anal. 2012, Article ID 965751 (2012). doi:10.1155/2012/96575

26. Sahin, A, Basarir, M: On the strong convergence of modified S-iteration process for asymptotically quasi-nonexpansive mappings in CAT(0) space. Fixed Point Theory Appl. 2013, Article ID 12 (2013). doi:10.1186/1687-1812-2013-12

27. Agarwal, RP, O'Regan, D, Sahu, DR: Iterative construction of fixed points of nearly asymptotically nonexpansive mappings. J. Nonlinear Convex Anal. 8(1), 61-79 (2007)

28. Guo, WP, Cho, YJ, Guo, W: Convergence theorems for mixed type asymptotically nonexpansive mappings. Fixed Point Theory Appl. 2012, Article ID 224 (2012). doi:10.1186/1687-1812-2012-224

29. Chidume, CE, Ofoedu, EU, Zegeye, H: Strong and weak convergence theorems for asymptotically nonexpansive mappings. J. Math. Anal. Appl. 280, 364-374 (2003)

30. Xu, HK: Existence and convergence for fixed points of mappings of asymptotically nonexpansive type. Nonlinear Anal., Theory Methods Appl. 16(12), 1139-1146 (1991)

31. Chang, SS, Cho, YJ, Zhou, H: Demi-closed principle and weak convergence problems for asymptotically nonexpansive mappings. J. Korean Math. Soc. 38, 1245-1260 (2001)

32. Browder, FE: Semicontractive and semiaccretive nonlinear mappings in Banach spaces. Bull. Am. Math. Soc. 74 660-665 (1968)

doi:10.1186/1687-1812-2013-122

Cite this article as: Chang et al.: Strong and $\Delta$-convergence for mixed type total asymptotically nonexpansive mappings in CAT(0) spaces. Fixed Point Theory and Applications 2013 2013:122.

\section{Submit your manuscript to a SpringerOpen ${ }^{\ominus}$ journal and benefit from:}

- Convenient online submission

- Rigorous peer review

- Immediate publication on acceptance

- Open access: articles freely available online

- High visibility within the field

- Retaining the copyright to your article 